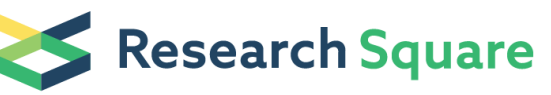

\section{Prenatal Diagnosis of Fetal Skeletal Dysplasia Using 3-Dimensional Computed Tomography: A Case Series}

\section{Miyoko Waratani ( $\sim$ mwara@koto.kpu-m.ac.jp )}

Kyoto Prefectural University of Medicine https://orcid.org/0000-0003-3353-6410

\section{Fumitake Ito}

Kyoto Prefectural University of Medicine

\section{Yukiko Tanaka}

Kyoto Prefectural University of Medicine

\author{
Aki Mabuchi \\ Kyoto Prefectural University of Medicine \\ Taisuke Mori \\ Kyoto Prefectural University of Medicine \\ Jo Kitawaki \\ Kyoto Prefectural University of Medicine
}

\section{Research}

Keywords: fetal skeletal dysplasia, fetal diagnosis, 3D-CT, shortening of long bones, gene expression

Posted Date: March 30th, 2020

DOI: https://doi.org/10.21203/rs.3.rs-19453/v1

License: (1) This work is licensed under a Creative Commons Attribution 4.0 International License. Read Full License 


\section{Abstract}

Background: Fetal skeletal dysplasia constitutes a complex group of systemic bone and cartilage disorders. Many fetal skeletal dysplasia phenotypes have indistinct definitions, making definitive prenatal diagnosis difficult. Fetal skeletal dysplasia is typically diagnosed using sonography; however, threedimensional computed tomography (3D-CT) also aids in making a prenatal diagnosis. This study aimed to examine the efficacy of 3D-CT for the prenatal diagnosis of fetal skeletal dysplasia by comparing the diagnostic accuracy of fetal sonography and 3D-CT.

Methods: Based on the suspicion of skeletal dysplasia based on ultrasound examination, we performed 3D-CT prenatally to confirm this diagnosis. In order to minimize exposure of the fetuses to radiation without compromising image quality, we used predetermined 3D-CT settings for volume acquisition.

Results: Sixteen fetuses were suspected to have fetal skeletal dysplasia based on ultrasonography findings. Of these, 15 were diagnosed with fetal skeletal dysplasia using 3D-CT. All 15 fetuses diagnosed with fetal skeletal dysplasia prenatally were confirmed postnatally to have fetal skeletal dysplasia. The definitive postnatal diagnosis (campomelic dysplasia) differed from the prenatal diagnosis (osteogenesis imperfecta) in only one infant. 14 cases (93.3\%) were diagnosed by an accyrate classification. Five infants had lethal skeletal dysplasia; one died in utero, and four died as neonates. We determined the appropriate delivery method for each infant based on the prenatal diagnosis.

Conclusion: 3D-CT is a valuable tool to augment ultrasound examination for the diagnosis of skeletal dysplasia because it can provide a more definitive diagnosis of the type of dysplasia, enabling better planning for resuscitation of the infant after birth.

Trial registration: University Hospital Medical Information Network (UMIN) Center trial registration number is UMIN000034744. Registered 1 October, 2018 - Retrospectively registered, https://upload.umin.ac.jp/cgi-open-bin/ctr_e/ctr_view.cgi?recptno=Roooo39610.

\section{Plain English Summary}

Fetal skeletal dysplasia is a disease of the bones and cartilage that develops prenatally. It is difficult to ascertain if fetal skeletal dysplasia is present before the baby is born. Fetal skeletal dysplasia is usually diagnosed using ultrasonography; however, three-dimensional computed tomography (3D-CT) also helps in making a diagnosis during pregnancy. Our study investigated whether 3D-CT could be helpful in the prenatal diagnosis of fetal skeletal dysplasia by comparing the diagnoses using fetal ultrasonography and 3D-CT with the diagnosis made at birth. In this study, we performed 3D-CT scans on 16 fetuses that were suspected to have fetal skeletal dysplasia based on ultrasonography findings. We used predetermined 3D-CT settings in order to minimize the radiation risk to the fetuses while still maintaining image quality. We diagnosed fetal skeletal dysplasia prenatally in 15 cases using 3D-CT. All 15 fetuses diagnosed with fetal skeletal dysplasia via 3D-CT were confirmed at birth to have fetal skeletal dysplasia although the type of dysplasia diagnosed was different in one case. Five infants had lethal skeletal 
dysplasia; one was stillborn, and four died shortly after birth. We concluded that 3D-CT is a valuable tool to augment an ultrasound examination for the diagnosis of skeletal dysplasia. With 3D-CT, the type of dysplasia can be more accurately diagnosed, and this information helps when making plans for the resuscitation of the infant after birth.

\section{Keywords}

fetal skeletal dysplasia, fetal diagnosis, 3D-CT, shortening of long bones, gene expression

\section{Background}

Fetal skeletal dysplasia (FSD) is a group of systemic bone and cartilage disorders that develop prenatally and may be detected by fetal ultrasonography. Because most cases of skeletal dysplasia involve the mutation of a single gene, a definitive diagnosis can be reached if this mutation is identified. Although the widespread use of fetal ultrasound imaging has improved the detection of FSD, prenatal diagnosis remains relatively difficult because of the rarity of the condition (approximately one of every 5,000 births) [1]. The diversity of the FSD phenotypes also contributes to the difficulty in diagnosing these disorders. FSDs comprise 436 diseases that are classified into 42 groups, according to the Nosology \& Classification of Genetic Skeletal Disorders: 2015 Revision [2]; however, the phenotypic definition of each condition is often unclear. It is important to make a definitive diagnosis to differentiate between lethal or nonlethal disorders in order to plan the most effective delivery method and the best postnatal treatment [3].

Three-dimensional computed tomography (3D-CT) is commonly used to diagnose cardiac atherosclerosis, cerebral aneurysms, and cerebral artery stenosis. This imaging technique creates better images of thromboses and vascular wall calcification in cerebral aneurysms compared to cerebral angiography. In 2004, Ruano et al. reported that fetal 3D-CT was a useful diagnostic method, complementary to ultrasonography, that may improve the accuracy of the prenatal diagnosis of skeletal disorders [4]. Considering both the clinical benefit of this method and the increased risk introduced by radiation, we elected to use low-dose 3D-CT for prenatal diagnosis in this study. The aim of this study was to compare the prenatal diagnosis and definitive postnatal diagnosis in patients with FSD in order to examine the efficacy of 3D-CT for the prenatal diagnosis of FSD.

\section{Methods}

\section{Study design}

A prospective study was conducted involving pregnant women whose pregnancy/childbirth was being managed in the Department of Obstetrics and Gynecology at Kyoto Prefectural University of Medicine. Fetuses were included in the study if they had a suspected bone abnormality based on routine ultrasound examinations. We recorded any family history of skeletal dysplasia and complications during pregnancy. 


\section{Materials}

Pregnant women whose pregnancies/childbirths were managed at our hospital and who met the following selection criteria were included as participants: (1) pregnancy at age 20 years or older at the time of consent, (2) gestational age $>26$ weeks of pregnancy but had not yet delivered, (3) ultrasonography showing a short fetal femur ( $\leq-2.5$ standard deviation of the reference of fetal measurements) or an image of a fracture, curvature, or deformity in a long bone, skull, rib, or spine, (4) the pregnant woman provided written informed consent to undergo 3D-CT.

\section{Sonographic examination}

We used Voluson E8 and E10 ultrasound systems (General Electric Healthcare, Tokyo, Japan) to screen for associated anomalies. All operators were experts in fetal sonography. First, we measured the fetus using a standard approach (biparietal diameter, abdominal circumference, and femur length) and identified fetuses that displayed femur length measurements below the $10^{\text {th }}$ percentile. We recommended 3D-CT scanning if the fetal femur or any other long bones were found to be shortened, regardless of whether or not they were abnormally shaped, indicating potential FSD.

\section{Three-dimensional computed tomography}

3D-CT (Aquilion RXL; TOSHIBA, Tokyo, Japan) was only performed after thoroughly explaining the effects of radiation exposure to the mother and fetus and receiving informed consent from the mother. In order to minimize exposure of the fetus to radiation without compromising image quality, the following predetermined settings were used for volume acquisition: 1) 1.00-mm thick scans with 1.0-mm intervals for $3.1 \mathrm{~s}$, 2) $120-\mathrm{mA}$ intensity, 3) $120-\mathrm{kV}$ voltage, 4) 0.5 -s rotation time, and 5) 1.438 pitch. Keeping these settings constant resulted in a median CT dose index volume of $2.9 \mathrm{mGy}$ (range: 1.9-4.9 mGy). We created a maximum intensity projection image from 3D-CT images for diagnosis, if necessary. Additional tests, such as magnetic resonance imaging (MRI) or amniotic chromosomal testing, were performed if required to determine fetal prognosis or to differentiate skeletal dysplasia from other diseases. Lethality was determined by the presence of a narrow thorax and lung hypoplasia based on ultrasonograms and MRI images.

\section{Postnatal examination}

Postnatally, we recorded the neonatal weight and length, and performed an X-ray examination to verify the shape of all bones. After genetic counseling, genetic testing was performed to confirm the diagnosis. Briefly, genomic DNA was extracted from the umbilical cord or the infant's blood.

\section{Results}

\section{Patient characteristics}


We identified 38 fetuses with a short femur, and based on sonography, 16 of these 38 fetuses had suspected FSD. We performed 3D-CT on these 16 fetuses. The mean maternal age was 32.6 (range: 2041) years, and the mean gestational age at the first findings of FSD was 24.4 (range: 15-36) weeks. The mean 3D-CT imaging period was 30.6 weeks' gestation, which is consistent with the period when CT testing is generally performed for prenatal diagnosis (approximately 30 weeks' gestation). Patient characteristics are presented in Table 1.

Table 1: Characteristics of 15 infants with SD

\begin{tabular}{|c|c|c|c|c|c|c|}
\hline Infant & $\begin{array}{c}\text { Maternal age } \\
\text { (years) }\end{array}$ & $\begin{array}{c}\text { GA at the first finding } \\
\text { of FSD (weeks) }\end{array}$ & $\begin{array}{l}\text { GA at delivery } \\
\text { (weeks) }\end{array}$ & $\begin{array}{c}\text { Delivery } \\
\text { mode }\end{array}$ & $\begin{array}{l}\text { Birth } \\
\text { weight } \\
\text { (g) }\end{array}$ & Prognosis \\
\hline 1 & 33 & 36 & 38 & $\mathrm{CS}$ & 2599 & alive \\
\hline 2 & 20 & 27 & 34 & $\mathrm{CS}$ & 2243 & alive \\
\hline 3 & 30 & 30 & 36 & $\mathrm{BE}$ & 1587 & 1 day \\
\hline 4 & 38 & 15 & 37 & CS (breech) & 2169 & 3 days \\
\hline 5 & 27 & 24 & 37 & VD & 2233 & 2 days \\
\hline 6 & 30 & 24 & 37 & $\mathrm{CS}$ & 2924 & alive \\
\hline 7 & 34 & 29 & 38 & VD & 3400 & alive \\
\hline 8 & 40 & 25 & 38 & VD & 2621 & alive \\
\hline 9 & 41 & 27 & 38 & CS (NRFS) & 2080 & alive \\
\hline 10 & 29 & 33 & 38 & VD & 2496 & alive \\
\hline 11 & 39 & 17 & 37 & $\mathrm{CS}$ & 2569 & alive \\
\hline 12 & 39 & 30 & 39 & CS (repeat) & 1843 & alive \\
\hline 13 & 38 & 18 & 37 & CS (breech) & 2866 & alive \\
\hline 14 & 25 & 16 & 27 & VD & - & IUFD \\
\hline 15 & 26 & 15 & 38 & $\begin{array}{c}\text { CS } \\
\text { (breech) }\end{array}$ & 1710 & 1 day \\
\hline
\end{tabular}


BE: breech extraction, CS: Cesarean section, GA: gestational age, IUFD: intrauterine fetal death, NRFS: non-reassuring fetal status, SD: skeletal dysplasia, VD: vaginal delivery

\section{Diagnostic accuracy}

Of the 16 fetuses that underwent 3D-CT, 15 were prenatally diagnosed with FSD. FSD was confirmed postnatally in all 15 of these fetuses. On the basis of prenatal 3D-CT, we suspected a chromosomal abnormality (trisomy 21 ) in the remaining fetus, which was confirmed postnatally.

\section{Classification of fetal skeletal dysplasia}

Prenatal imaging data, postnatal findings, and pre- and postnatal diagnosis are presented in Table 2 . The specific diagnoses were as follows: osteogenesis imperfecta $(\mathrm{OI}, \mathrm{n}=3)$, thanatophoric dysplasia $(T D, n=2)$, achondroplasia/hypochondroplasia $(\mathrm{ACH} / \mathrm{HCH}, \mathrm{n}=5)$, hypophosphatasia ( $\mathrm{HPP}, \mathrm{n}=2)$, campomelic dysplasia $(C D, n=1)$, Desbuquois dysplasia $(n=1)$, and Creveld syndrome $(n=1)$. The two infants with HPP were siblings.

Table 2: Comparison between pre- and postnatal findings and diagnosis 


\begin{tabular}{|c|c|c|c|c|}
\hline \multirow[t]{2}{*}{ Infant } & \multicolumn{2}{|l|}{ Prenatal } & \multicolumn{2}{|l|}{ Postnatal } \\
\hline & findings & diagnosis & findings & diagnosis \\
\hline \multirow[t]{3}{*}{1} & Shortening and bowing of long & OI & Shortening and bowing of long bones, & OI \\
\hline & bones, & & Rib fractures & \\
\hline & Rib fractures & & & \\
\hline \multirow[t]{2}{*}{2} & Shortening and bowing of long & OI & Shortening and fractures flong bones & OI \\
\hline & bones, hydramnios & & & \\
\hline \multirow[t]{3}{*}{3} & Shortening and bowing of long & OI & Shortening and bowing of long bones, Facial & OI \\
\hline & bones, Narrow thorax, & & dysmorphy, Rib fractures, Narrow thorax & \\
\hline & Megalencephaly & & & \\
\hline \multirow[t]{3}{*}{4} & Shortening of long bones, Narrow & TD & Rhyzomelic shortening of long bones, Bowing of long & $\mathrm{TD}$ \\
\hline & thorax, Megalencephaly & & bones, Megalencephaly, Narrow thorax, & \\
\hline & & & Platyspondylia & \\
\hline \multirow[t]{4}{*}{5} & Shortening and bowing of long & $\mathrm{TD}$ & Shortening and bowing of long bones, & TD \\
\hline & bones, & & Trident hand, Megalencephaly, Frontal bossing, & \\
\hline & Narrow thorax, Megalencephaly, & & Narrow thorax, Platyspondylia & \\
\hline & Hydramnios & & & \\
\hline \multirow[t]{2}{*}{6} & Shortening of long bones, Narrow & $\mathrm{ACH}$ or & Shortening of long bones, Narrow thorax & $\mathrm{ACH}$ \\
\hline & thorax & $\mathrm{HCH}$ & & \\
\hline \multirow[t]{2}{*}{7} & Shortening of long bones, Narrow & $\mathrm{ACH}$ & Shortening of long bones, Narrow thorax & $\mathrm{ACH}$ \\
\hline & thorax & & & \\
\hline \multirow[t]{2}{*}{8} & Shortening of long bones, Narrow & $\mathrm{ACH}$ & Shortening of long bones, Frontal bossing, Narrow & $\mathrm{ACH}$ \\
\hline & thorax, Trident hand & & thorax, Trident hand & \\
\hline \multirow[t]{3}{*}{9} & Shortening of long bones, Narrow & $\mathrm{ACH}$ & Shortening of long bones, Frontal bossing, Narrow & $\mathrm{ACH}$ \\
\hline & thorax, Trident hand & & thorax, Trident hand & Trisomy 21 \\
\hline & & & Facial dysmorphy of Down syndrome & \\
\hline \multirow[t]{2}{*}{10} & Shortening of long bones & $\mathrm{ACH}$ or & Shortening of long bones, Frontal bossing & $\mathrm{HCH}$ \\
\hline & & $\mathrm{HCH}$ & & \\
\hline
\end{tabular}

11 Shortening of long bones, bowing

OI Bowing of long bones, Club feet, Hypoplastic

CD 
of long bones, Club feet,

Hypoplastic scapulae

12

Dislocation of elbow and knee,

Club feet

disorder scapulae, Rib fractures,

Ambiguous genitalia, Facial dysmorphy

Dislocation of shoulder, elbow,

Desbuquois

disorder

Facial dysmorphy, Scoliosis, brevicollis

13

Shortening of long bones,

Elis-van

Shortening of long bones, Narrow thorax,

Elis-van

Hyperdactylia

Creveld

Hyperdactylia

Creveld

CHD (AVSD, single atrium)

syndrome

CHD (AVSD, single atrium)

syndrome
HPP

14 Shortening of long bones, Narrow

HPP

HPP

15

Shortening of long bones, Narrow

HPP

Shortening of long bones, Narrow thorax,

thorax, hydramnios

Hypoplasia of lung

Low levels of serum ALP

ACH: achondroplasia, AVSD: atrial ventricular septal defect, CD: campomelic dysplasia, CHD: congenital heart disease, $\mathrm{HCH}$ : hypochondroplasia, HPP: hypophosphatasia, Ol: osteogenesis imperfecta, TD: thanatophoric dysplasia

The pre- and postnatal diagnoses differed in one infant diagnosed with OI prenatally and CD postnatally. In this case, the 3D-CT fetal skeleton findings (long bone shortening and mild curvature with scapular hypoplasia; Figure 1) were the same as the postnatal findings. However, we identified ambiguous genitalia, one of the characteristic findings of $C D$, in the postnatal period.

\section{Lethality}

Of the five fetuses with poor prognoses, one had OI, two had TD, and two had HPP. One of the fetuses with HPP suffered intrauterine fetal death at 27 weeks' gestation, while the remaining four infants died within two days of birth. We identified lethality in the prenatal period in all five fetuses.

\section{Trisomy 21 and achondroplasia}

Infant 8 was diagnosed prenatally with $\mathrm{ACH}$ based on ultrasound findings of shortened long bones, a narrow thorax, and a trident hand (Figure 2). After birth, the characteristic trisomy 21 facial features were noted, and the results of chromosomal testing (G-band; 47, XY, +21) confirmed trisomy 21. FGFR3 genetic analysis also indicated a G1193A mutation in G380, indicating that the infant also had ACH.

\section{Postnatal genetic testing}


In infant 12, we identified dislocation of the knee and elbow, as well as clubfeet, based on sonography and 3D-CT, leading us to suspect Desbuquois dysplasia (Figure 3). Postnatal genetic testing confirmed our diagnosis of Desbuquois dysplasia (type 1) due to a mutation of the 613165 calcium-activated nucleotidase 1 (CANT1) gene.

The two infants with HPP (infants 14 and 15) had the same parents. The first child exhibited marked long bone shortening and a soft, deformed skull based on ultrasonography at 18 weeks' gestation, which was suggestive of fetal HPP. Therefore, we measured maternal alkaline phosphatase (ALP) levels, which were low. At 27 weeks' gestation, the child suffered from intrauterine fetal death. After the child was stillborn, autopsy imaging was performed. The CT images also indicated a likely diagnosis of HPP. Genetic testing of the placental tissue revealed a compound heterozygote with mutations in two tissue-nonspecific alkaline phosphatase genes (ALPL; p.K224E, c.1559delT), and confirmed our diagnosis of HPP. The parents' second child exhibited severe shortening of all four limbs at 15 weeks' gestation, with 3D-CT revealing findings typical of severe perinatal HPP (Figure 4), with hardly any ossification. At 38 weeks' gestation, the infant was delivered via Cesarean section but suffered neonatal death at 1 day of age.

\section{Discussion}

Prenatal diagnosis of skeletal dysplasia is typically based on sonographic and radiological findings, and 3D-CT may be a valuable imaging tool complementary to ultrasonography for the diagnosis of skeletal dysplasia. However, it is necessary to select the appropriate settings to minimize the degree of fetal irradiation without compromising image quality [5]. In this study, we showed that when 3D-CT was used along with fetal ultrasonography, the prenatal diagnosis and definitive postnatal diagnosis differed in only one infant. This infant was diagnosed prenatally with FSD and postnatally with trisomy 21 . However, when sonography was used without 3D-CT, prenatal diagnoses differed from definitive postnatal diagnoses in three infants. In addition, the pre-and postnatal classifications of the specific form of FSD differed in one infant. In this infant, the prenatal 3D-CT findings were the same as the postnatal X-ray findings. However, we did not identify ambiguous genitalia in the infant (a characteristic finding of CD) [6] on the fetal ultrasound examination. Thus, we diagnosed CD based on the postnatal physical findings.

Fetal bone 3D-CT (as an auxiliary test for ultrasound examinations) has high diagnostic accuracy when it is performed from 26 weeks' gestation onward. The detection of abnormal findings outside the skeletal system on sonography can greatly affect the diagnosis $[7,8]$. For an accurate diagnosis of FSD, both sonography and 3D-CT appear to be useful methods. In some cases, it can be difficult to differentiate skeletal dysplasia from a chromosomal abnormality. Of the 16 infants that underwent 3D-CT in the present study, only one was suspected of having a chromosomal abnormality. In this case, skeletal dysplasia was suspected based on ultrasonography findings, and the results of 3D-CT suggested trisomy 21. Postnatal chromosomal testing was performed, resulting in a final diagnosis of trisomy 21 and the exclusion of this infant from the present study. Some cases can be complicated, with concomitant skeletal dysplasia and a chromosome aberration. In particular, combinations of ACH and trisomy 21 have been reported $[9,10]$. ACH is a mild type of dysplasia in the group of FGFR3 abnormalities, where the parts 
that grow become hypoplastic due to endochondral ossification, which can be difficult to differentiate from trisomy 21 [9,10]. In 2001 [11] and 2014 [12], it was reported that femoral shortening during the second trimester is associated with a high relative risk of chromosomal abnormalities, particularly trisomy 21. If fetal femoral shortening is observed, it is important to consider the possibility of conditions, such as chromosomal abnormalities, fetal growth restriction, and various other anomalies. Attention should also be paid to the presence of abnormal findings outside of the skeletal system (e.g., abnormalities in cardiac structure, the nervous/gastrointestinal system, and facial features) to reach a differential diagnosis. When making a prenatal diagnosis, it is important to bear in mind that cases of concomitant skeletal dysplasia and chromosomal abnormalities are possible, albeit rare.

Desbuquois dysplasia is a severe autosomal recessive disorder that belongs to the multiple dislocation group [13]. It is characterized by shortened long bones, joint laxity, pre- and postnatal growth retardation, and progressive scoliosis. In the case of infant 12, the mother's first child also had skeletal dysplasia (congenital dislocation of the knees) that had been identified after birth. Although this child had been diagnosed with Larsen syndrome, it had not been identified prenatally by sonography/3D-CT nor confirmed via postnatal genetic testing. The mother's other child (infant 12), on the other hand, received a prenatal ultrasound examination and 3D-CT, and was diagnosed with Desbuquois dysplasia, rather than Larsen syndrome. Postnatal genetic testing led to a definitive diagnosis of Desbuquois dysplasia (type 1) due to a mutation in the CANT1 gene [14,15]. Conversely, Larsen syndrome is due to a mutation in the 603381 Filamin B gene. This example demonstrates that an accurate clinical diagnosis is important to achieve a definitive diagnosis via postnatal molecular evaluation. Importantly, prenatal 3D-CT imaging is a valid diagnostic method that may be employed in such cases.

HPP is a condition in which tissue non-specific ALP loss or decrease causes defective bone mineralization and abnormal tooth mineralization [16]. Many cases of HPP are inherited in an autosomal recessive manner, whereas others are autosomal dominant. HPP can be classified into the following six clinical forms, which vary based on severity [17]: 1) perinatal severe, 2) perinatal benign, 3) infantile, 4) childhood, 5) adult, and 6) odontohypophosphatasia. If parents have a child with HPP, they should be given genetic counseling with respect to future pregnancies, and the couple can decide whether to undergo carrier detection testing. If both the mother and father are carriers, the child has a $25 \%$ probability of being homozygous. Our fatal perinatal case appeared to be a severe case. The symptoms of perinatal cases appear early, resulting in intrauterine fetal death [18], stillbirth, or neonatal death.

Compared to normal fetuses, those with FSD are more likely to be in an abnormal fetal position owing to differences in physique, restricted posture, limited joint range of motion, and fractures [19]. In cases of OI, it can be difficult to prevent intrauterine fractures as they can be caused by fetal movement (Figure 5). Studies have shown that, in cases of Ol, performing a Cesarean delivery does not increase the frequency of fetal bone fractures compared that with vaginal delivery [20]. Moreover, in cases of the lethal type of Ol, fetal survival does not appear to be affected by delivery mode (Cesarean vs. vaginal delivery). For other types of skeletal dysplasias, the mode of delivery is often selected based on whether or not the condition is lethal. When the disease is diagnosed as lethal, a vaginal delivery may be selected even if the 
fetus is breech. However, in cases with marked head enlargement, a Cesarean section is required regardless of whether or not the case is fatal, owing to the potential difficulty in passing through the mother's pelvis. It is important for the parents, obstetrician, and pediatrician to discuss prior to delivery the plan for the resuscitation of the infant after birth. Accurate prenatal diagnosis is required to determine whether or not the case is fatal, and the degree of severity.

When considering the risk of radiation exposure, CT examination has not historically been the preferred method for pregnant women. However, in recent years, high quality images adequate for a diagnosis have been made by low-dose 3D-CT, reducing the level of radiation to which the mother and fetus are exposed. The strength of this study was the accuracy of the prenatal diagnosis by 3D-CT scan in combination with fetal sonography. The limitation of this study is the relatively small sample size. This should be increased in future studies to improve the power of the results. Continuation of this study is important in order to advance the diagnosis of fetal skeletal dysplasias.

\section{Conclusions}

In conclusion, 3D-CT is a valuable tool for augmenting ultrasound examinations in the diagnosis of FSD. The improved diagnostic skill of sonography is important in cases of suspected FSD, but 3D-CT imaging is indispensable for an accurate diagnosis and classification.

\section{Declarations}

Ethics approval and consent to participate: The institutional review boards of Kyoto Prefectural University of Medicine (ERB-C-991) and all the participating hospitals approved the protocol. Written informed consent was obtained from all patients before registration, in accordance with the Declaration of Helsinki. Results of the study will be disseminated via publication in peer-reviewed journals.

Consent for publication: Not applicable.

Availability of data and materials: The datasets generated and/or analyzed during the current study are available in the University Hospital Medical Information Network (UMIN). (URL:

https://upload.umin.ac.jp/cgi-open-bin/ctr_e/ctr_view.cgi?recptno=R000039610)

Competing interests: The authors declare that they have no competing interests.

Funding: This research received no specific grant from any funding agency in the public, commercial or not-for-profit sectors.

Authors' contributions: The author contributions are as follows: MW conceived and designed the study, wrote the protocol manuscript, and analyzed and interpreted the data. FI, YT, AM, and TM collected the study data. JK provided guidance regarding the study design and ethical approval. All authors read and approved the final manuscript. 
Acknowledgments: We thank the patients and their families involved in this study. We would like to thank Editage (www.editage.com) for English language editing.

\section{Abbreviations}

FSD, fetal skeletal dysplasia; 3D-CT, three-dimensional computed tomography; MRI, magnetic resonance imaging; Ol, osteogenesis imperfecta; TD, thanatophoric dysplasia; $\mathrm{ACH} / \mathrm{HCH}$, achondroplasia/hypochondroplasia; HPP, hypophosphatasia; CD, campomelic dysplasia; CANT1, calcium-activated nucleotidase 1; ALP, alkaline phosphatase

\section{References}

(1) Chen C, Jiang Y, Xu C, Liu X, Hu L, Xiang Y, et al. Skeleton genetics: a comprehensive database for genes and mutations related to genetic skeletal disorders. Database (Oxford). 2016:2016:baw127.

(2) Bonafe L, Comier-Daire V, Hall C, Lachman R, Mortier G, Mundlos S, et al. Nosology and classification of genetic skeletal disorders: 2015 revision. Am J Med Genet A. 2015;167A:2869-92.

(3) Krakow D, Lachman RS, Rimoin DL. Guidelines for the prenatal diagnosis of fetal skeletal dysplasias. Genet Med. 2009;11:127-33.

(4) Ruano R, Molhi M, Roume J, Ville Y. Prenatal diagnosis of fetal skeletal dysplasias by combining twodimensional and three-dimensional ultrasound and intrauterine three-dimensional helical computer tomography. Ultrasound Obstet Gynecol. 2004;24:134-40.

(5) Macé G, Sonigo P, Cormier-Daire V, Aubry MC, Martinovic J, Elie C, et al. Three-dimensional helical computed tomography in prenatal diagnosis of fetal dysplasia. Ultrasound Obstet Gynecol. 2013;42:1618.

(6) Jain V, Sen B. Campomelic dysplasia. J Pediatr Orthop B. 2014;23:485-8.

(7) Adler-Levy Y, Yagel S, Nadjari M, Bar-ziv Y, Simanovsky N, Hiller N. Use of low dose computed tomography with $3 \mathrm{D}$ reconstructions for the prenatal evaluation of suspected skeletal dysplasia. Isr Med Assoc J. 2015;17:42-6.

(8) Cassart M, Massez A, Cos T, Tecco L, Thomas D, Van Regemorter N, et al. Contribution of threedimensional computed tomography in the assessment of fetal skeletal dysplasia. Ultrasound Obstet Gynecol. 2007;29:537-43.

(9) Kaga A, Murotsuki J, Kamimura M, Kimura M, Saito-Hakoda A, Kanno J, et al. Association of achondroplasia with Down syndrome: difficulty in prenatal diagnosis by sonographic and 3-D helical computed tomographic analysis. Congenit Anom (Kyoto). 2015;55:116-20. 
(10) de Azevedo Moreira LM, Matos MA, Schiper PP, Carvalho AF, Gomes IC, Rolemberg JC, et al. Cooccurrence of achondroplasia and Down syndrome: Genotype/phenotype association. Birth Defects Res A Clin Mol Teratol. 2010;88:228-31.

(11) Smith-Bindman R, Hosmer W, Feldstein VA, Deeks JJ, Goldberg JD. Second-trimester ultrasound to detect fetuses with Down syndrome: a meta-analysis. JAMA. 2001;285:1044-55.

(12) Mathiesen JM, Aksglaede L, Skibsted L, Petersen OB, Tabor A, Danish Fetal Medicine Study Group. Outcome of fetuses with short femur length detected at second-trimester anomaly scan: a national survey. Ultrasound Obstet Gynecol. 2014;44:160-5.

(13) Huber C, Oulès B, Bertoli M, Chami M, Fradin M, Alanay Y, et al. Identification of CANT1 mutations in Desbuquois dysplasia. Am J Hum Genet. 2009;85:706-10.

(14) Inoue S, Ishii A, Shirotani G, Tsutsumi M, Ohta E, Nakamura M, et al. Case of Desbuquois dysplasia type 1: potentially lethal skeletal dysplasia. Pediatr Int. 2014;56:26-9.

(15) Laccone F, Schoner K, Krabichler B, Kluge B, Schwerdtfeger R, Schulze B, et al. Desbuquois dysplasia type 1 and fetal hydrops due to novel mutations in the CANT1 gene. Eur J Hum Genet. 2011;19:1133-7.

(16) Suzumori N, Mornet E, Mizutani E, Obayashi S, Ozaki Y, Sugiura-Ogasawara M. Prenatal diagnosis of familial lethal hypophosphatasia using imaging, blood enzyme levels, chorionic villus sampling and archived fetal tissue. J Obstet Gynaecol Res. 2011;37:1470-3.

(17) Nakamura-Utsunomiya A, Okada S, Hara K, Miyagawa S, Takeda K, Fukuhara R, et al. Clinical characteristics of perinatal lethal hypophosphatasia: a report of 6 cases. Clin Pediatr Endocrinol. 2010;19:7-13.

(18) Tongsong T, Pongsatha S. Early prenatal sonographic diagnosis of congenital hypophosphatasia. Ultrasound Obstet Gynecol. 2000;15: 52-5.

(19) Van Dijk FS, Sillence DO. Osteogenesis imperfecta: clinical diagnosis, nomenclature and severity assessment. Am J Med Genet A. 2014;164A:1470-81.

(20) Bellur S, Jain M, Cuthbertson D, Krakow D, Shapiro JR, Steiner RD, et al. Cesarean delivery is not associated with decreased at-birth fracture rates in osteogenesis imperfecta. Genet Med. 2016;18:570-6.

\section{Figures}




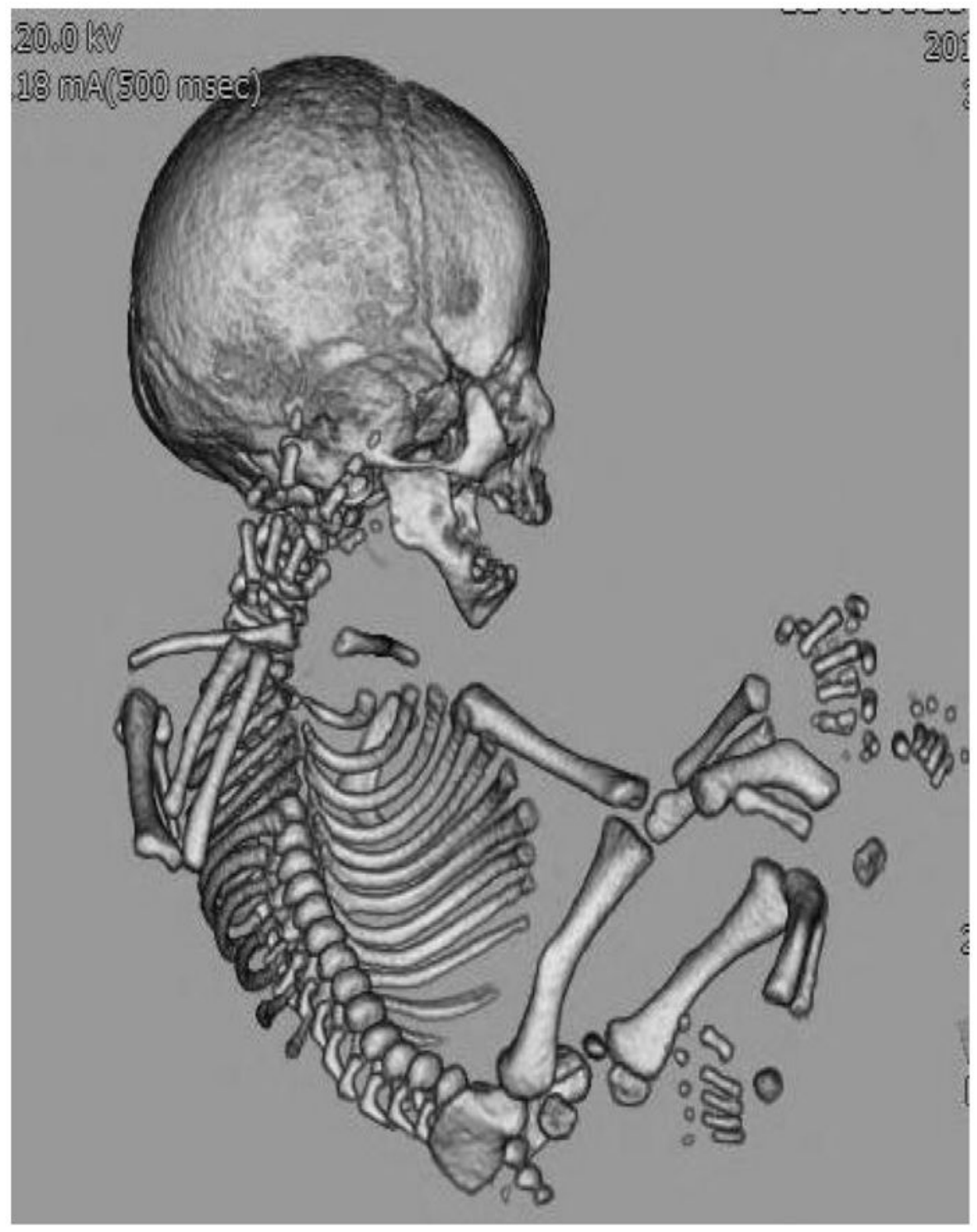

\section{Figure 1}

Fetal 3-dimensional computed tomography imaging of Infant 11: A case of campomelic dysplasia with a postnatal diagnosis that was different from the prenatal diagnosis 


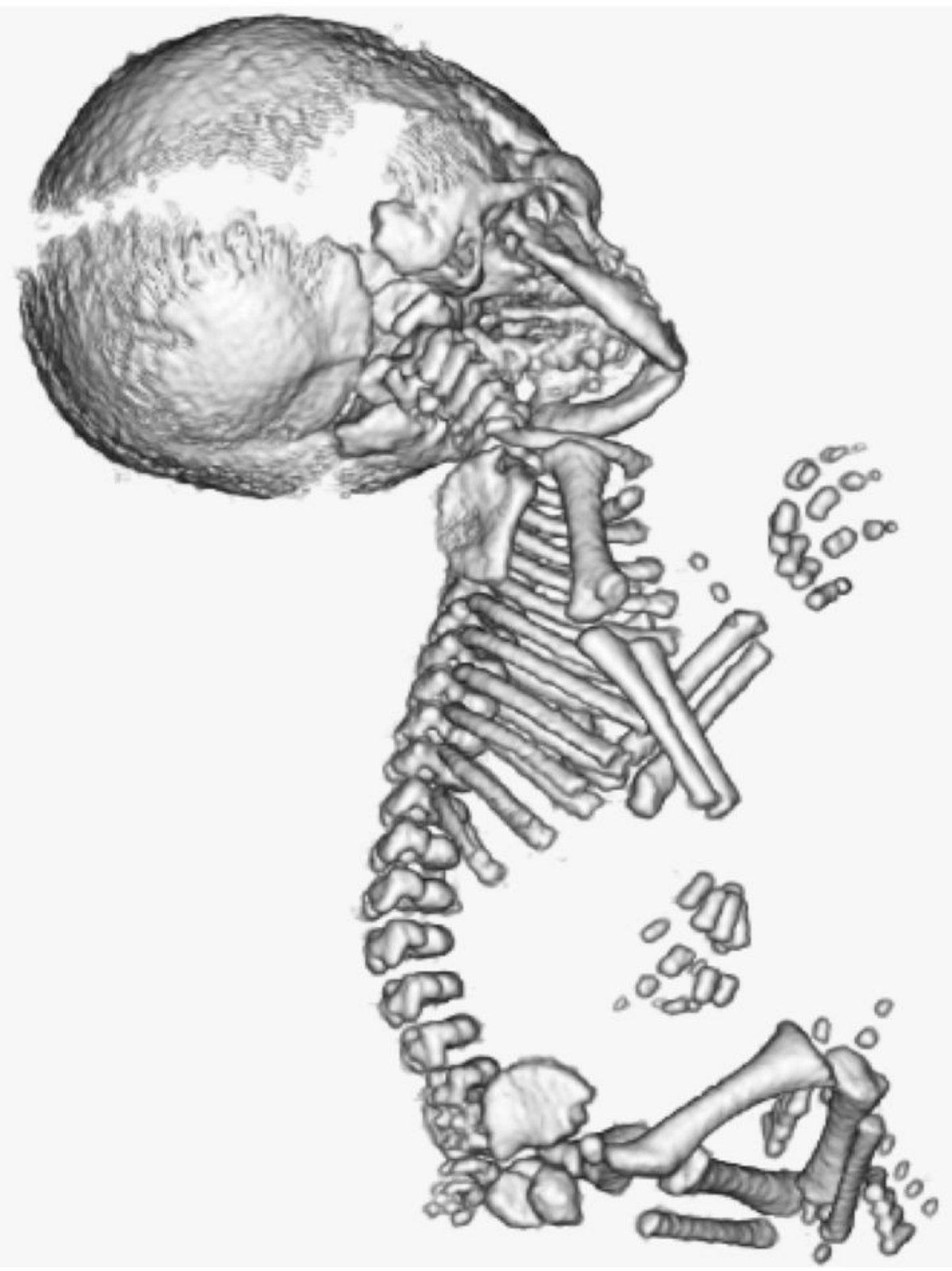

\section{Figure 2}

Fetal 3-dimensional computed tomography imaging of Infant 8: A case of achondroplasia with trisomy 21 


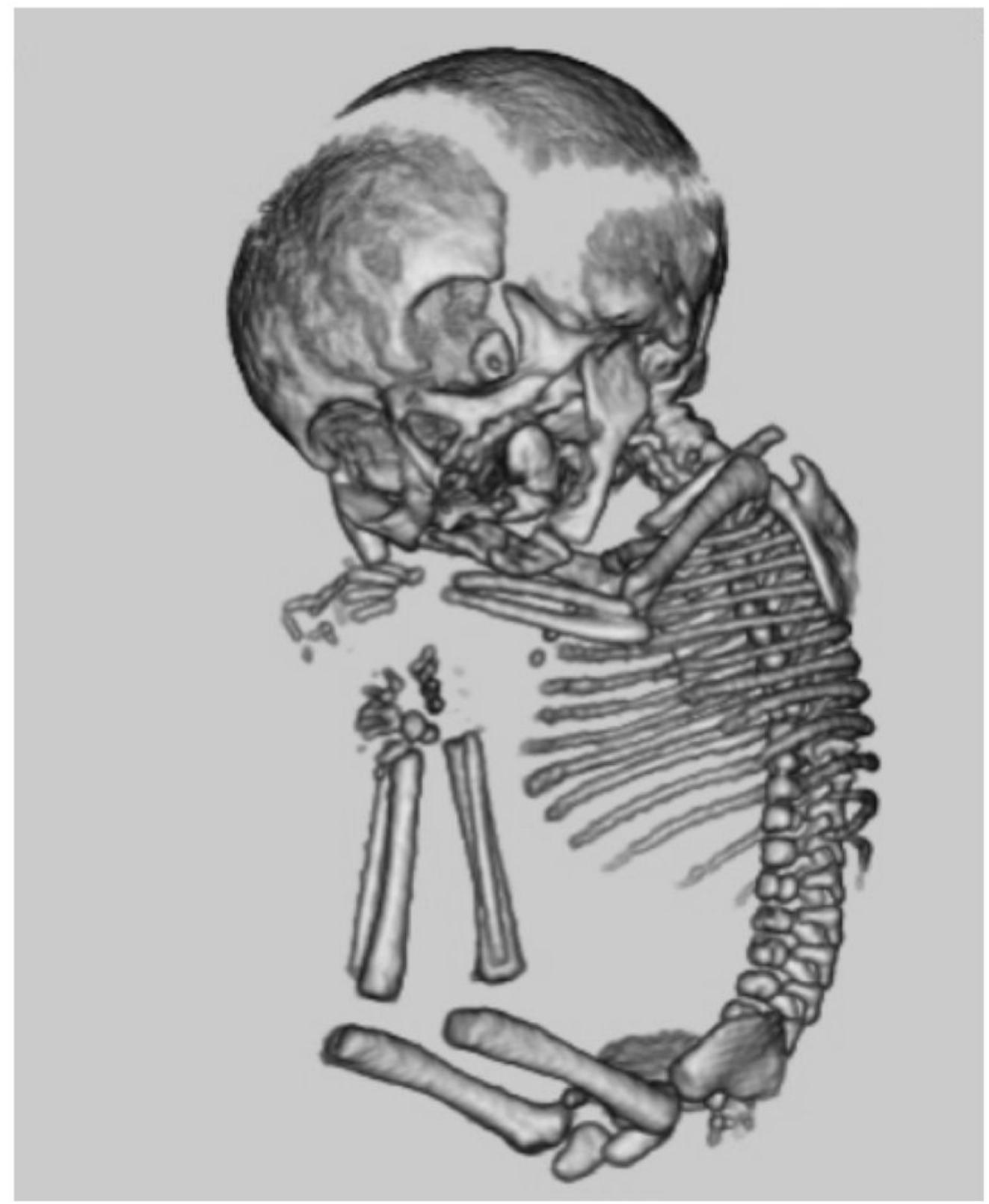

Figure 3

Fetal 3-dimensional computed tomography imaging of Infant 12: A case of Desbuquois dysplasia 


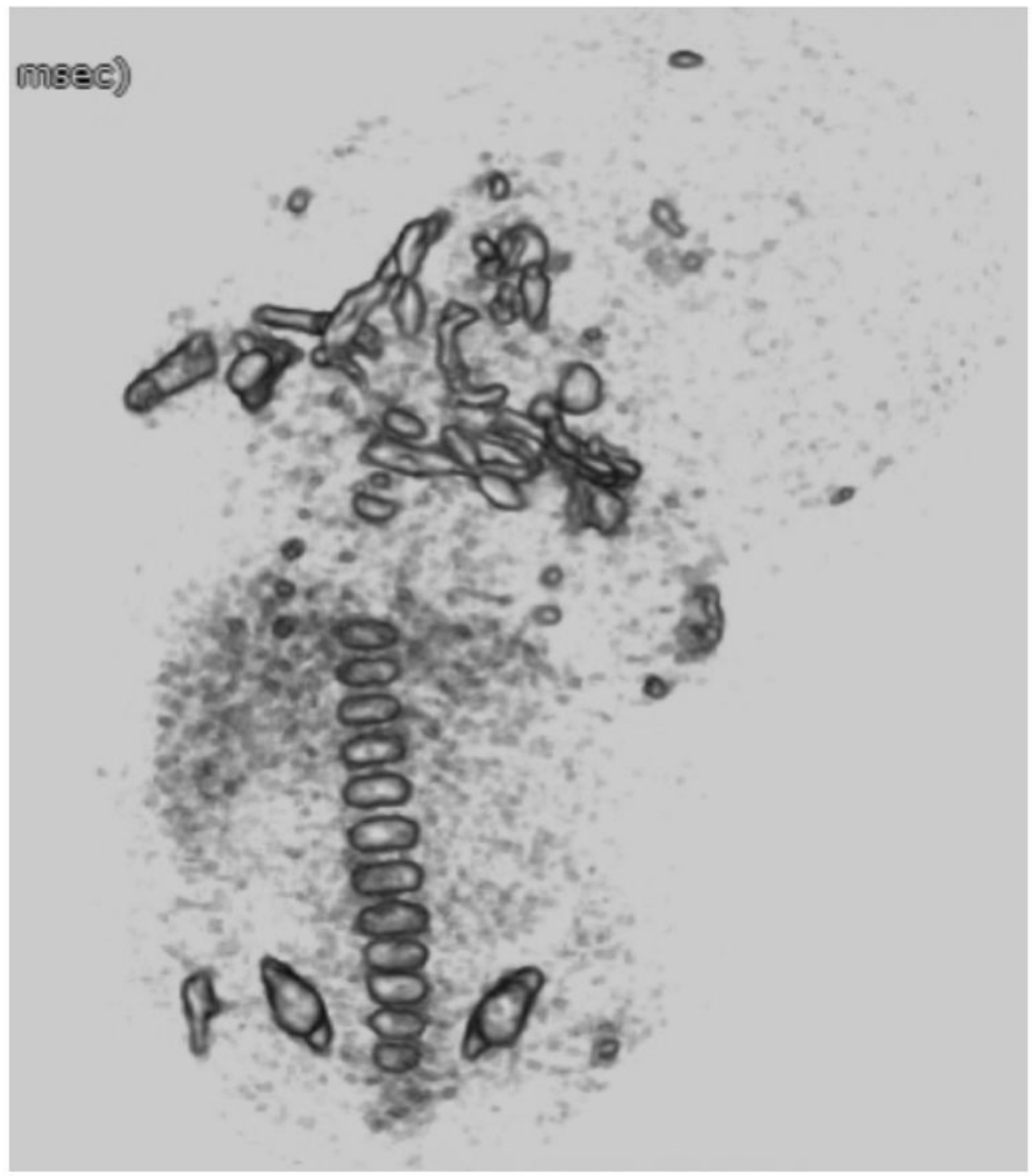

Figure 4

Fetal 3-dimensional computed tomography imaging of Infant 15: A case of hypophosphatasia 


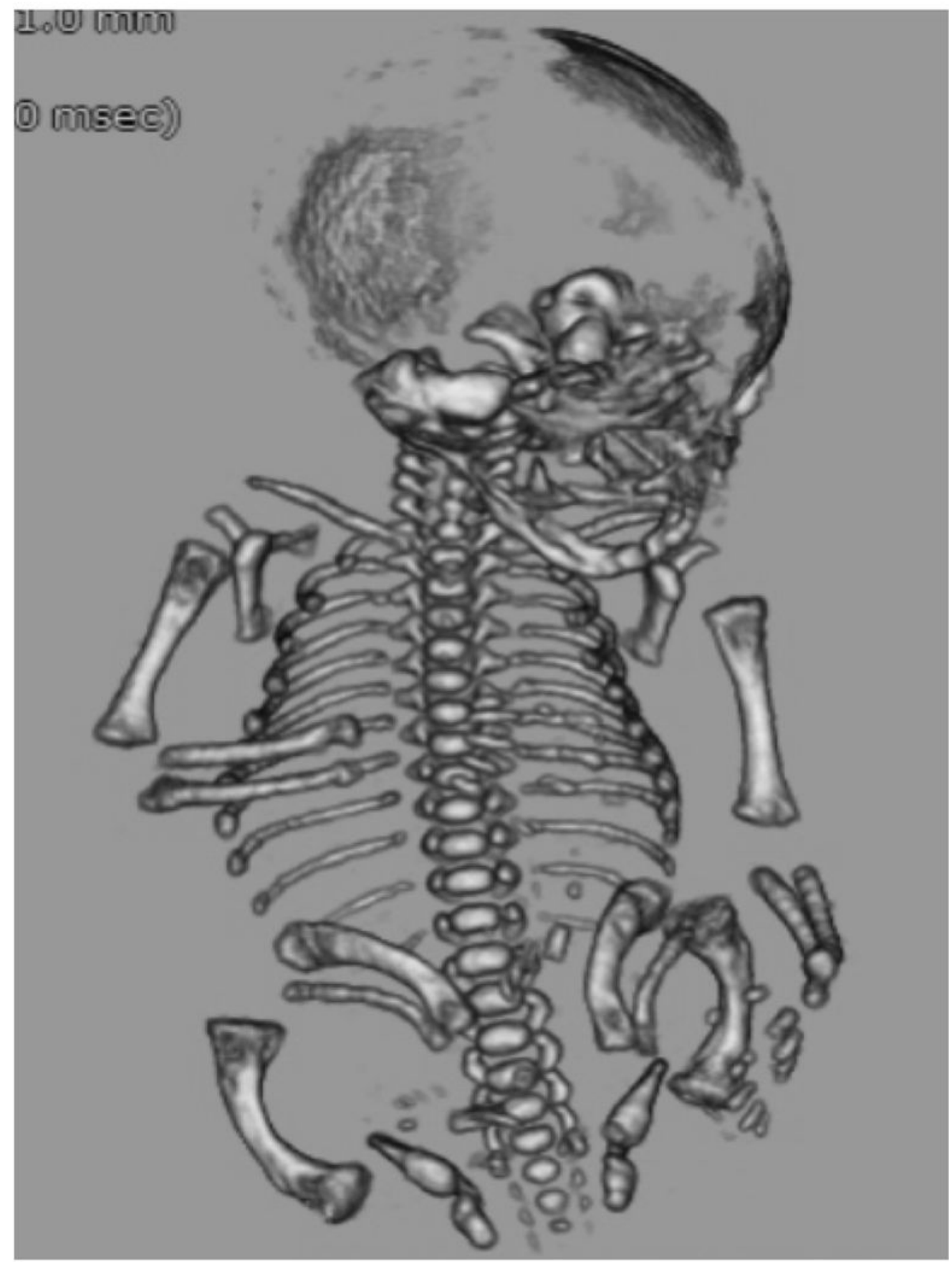

Figure 5

Fetal 3-dimensional computed tomography imaging of Infant 2: A case of osteogenesis imperfecta 